\title{
A DESCRIPTIVE STUDY OF THE EMERGENCY LAPAROTOMIES AT A GOVERNMENT TEACHING HOSPITAL
}

\author{
Khalid Muqueem ${ }^{1}$, Gowreesh Meti ${ }^{2}$
}

1 Associate Professor, Department of Surgery, Vijayanagara Institute of Medical Sciences, Ballari, Karnataka.

2Junior Resident, Department of Surgery, Vijayanagara Institute of Medical Sciences, Ballari, Karnataka.

\begin{tabular}{l} 
ABSTRACT \\
\hline BACKGROUND \\
Although, emergency laparotomy is a common surgical procedure performed routinely, yet outcome data and postoperative care \\
data is very less documented. Therefore, we undertook this systematic study of emergency laparotomies performed in our \\
Government Teaching Hospital.
\end{tabular}

\section{MATERIALS AND METHODS}

This is a Descriptive study of 137 consecutive emergency laparotomies, which were performed in our Government Teaching Hospital between 01 January 2015 and 31 December 2016. The clinical presentations, indications, intraoperative findings and complications after emergency laparotomies were properly documented and tabulated.

\section{RESULTS}

Patients who underwent emergency laparotomy presented with the symptoms of acute abdomen (78.9\%) or with history of trauma. The majority of cases who presented with acute abdomen were having gastrointestinal perforation (74\%). Next common aetiology was found to be intestinal obstruction (20\%). $89 \%$ of the laparotomies due to trauma were of blunt trauma type and the rest were of penetrating type. On examination, the most common clinical features were found to be abdominal tenderness (89.7\%), distension of abdomen (78.1\%), tachycardia (77.4\%), guarding (69.3\%) and hypotension (9.5\%). The age group of the majority of the patients was in the 20 - 50 years range. Nearly half (47.4\%) of the patients were taken for surgery within the first 24 hours of their arrival into the hospital. About $56.2 \%$ of patients developed postoperative complications, of which the most common was wound infection (27\%). After emergency laparotomies, 20 patients $(14.6 \%)$ died in the postoperative period during the initial hospital stay. Mortality was maximum in the elderly age group and in patients with large bowel perforation and in patients having extensive bowel gangrene due to mesenteric ischaemia. In trauma group, severe haemorrhagic shock and major organ or multiple solid organ injuries and/or great vessel injury were the mortal causes.

\section{CONCLUSION}

'Acute Abdomen' is the most common indication for emergency laparotomies. Among these cases of 'Acute Abdomen,' perforation peritonitis is the most common indication for a laparotomy. Although, the most common perforation in our study was duodenal perforation, the diagnosis-specific mortality in 'Acute Abdomen' cases was higher when the aetiology was large bowel perforation or mesenteric ischaemia. Time elapsed between presentation to the Emergency Room (ER) and surgical intervention had a bearing on the outcome.

\section{KEYWORDS}

Laparotomy, Peritonitis, Acute Abdomen, Intestinal Obstruction, Intestinal Perforation, Blunt Trauma.

HOW TO CITE THIS ARTICLE: Muqueem K, Meti G. A descriptive study of the emergency laparotomies at a government teaching hospital. J. Evolution Med. Dent. Sci. 2018;7(10):1194-1198, DOI: 10.14260/jemds/2018/273

\section{BACKGROUND}

An exploratory laparotomy is needed when the need for operation is definitely felt, but a definitive diagnosis is difficult or in some cases not possible until the opening of the abdomen. In cases of crushing or bruising of the abdomen or in cases due to injuries due to penetrating trauma, and in those presenting with 'acute abdomen' exploratory laparotomy is imperative to arrive at the diagnosis and also to treat the case.[1] Such surgeries are usually done on emergency basis due to possibility of high mortality, if the

'Financial or Other Competing Interest': None.

Submission 02-02-2018, Peer Review 19-02-2018,

Acceptance 21-02-2018, Published 05-03-2018.

Corresponding Author:

Dr. Khalid Muqueem,

C/o. Department of Surgery,

Vijayanagara Institute of Medical Sciences,

Ballari-583104, Karnataka, India.

E-mail:drkhalidmuqueem@yahoo.com

DOI: $10.14260 /$ jemds $/ 2018 / 273$

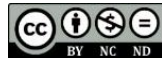

causative factor is not dealt with due to ongoing injury and/or actual or potential sepsis.

'Acute abdomen' refers to a clinical presentation with signs of symptoms of abdominal pain and tenderness, which often requires emergency surgical therapy.[2]

When a patient presents with 'Acute Abdomen,' a decision to proceed with laparotomy becomes very crucial, because any delay can be life-threatening. Even in such emergency settings, necessary investigations should be compulsorily done (if patient is stable) to arrive at a provisional diagnosis.

The organ involved in the disease, time elapsed from the time of onset of symptoms to the time of definitive surgery, condition of the patient, comorbidities, complications of anaesthesia and quality of care in the postoperative period are all important factors which can affect the outcome of the patients. ${ }^{[3]}$

\section{MATERIALS AND METHODS}

This is a hospital-based descriptive study, where all the patients who underwent Emergency Laparotomy in a 
Government Medical College, Teaching Hospital, were included in the study. The data was collected from our hospital records of 2 years, i.e. from 01 January 2015 to 31 December 2016. Patients who underwent Elective Laparotomy were excluded from this study.

\section{RESULTS}

This study is of 137 consecutive emergency laparotomy cases of all units of Department of Surgery. Out of these 137 cases, 29 cases $(21.2 \%)$ were done for trauma (both blunt and penetrating) and the rest (78.8\%) were done due to "Acute Abdomen." Majority of cases were in the 21 - 50 years of age group (70\%) and only 41 patients (29.9\%) were females and rest were males.

Vast majority (89.6\%) of the trauma laparotomies were of blunt injury type and the rest were of penetrating type. In 11 $(37.9 \%)$ trauma cases, associated injuries other than injuries in the abdomen were found.

History of substance abuse like alcoholism, smoking of more than 5 beedis/ cigarettes per day and intravenous drug abuse was present in $51(37.2 \%)$ of the patients. 18 patients $(13.1 \%)$ had the history of previous laparotomy. About $56.9 \%$ cases were having various comorbidities, requiring frequent medical follow-up and taking of regular medications.

Clinical Features of the Patients who later required Emergency Laparotomies are as follows-

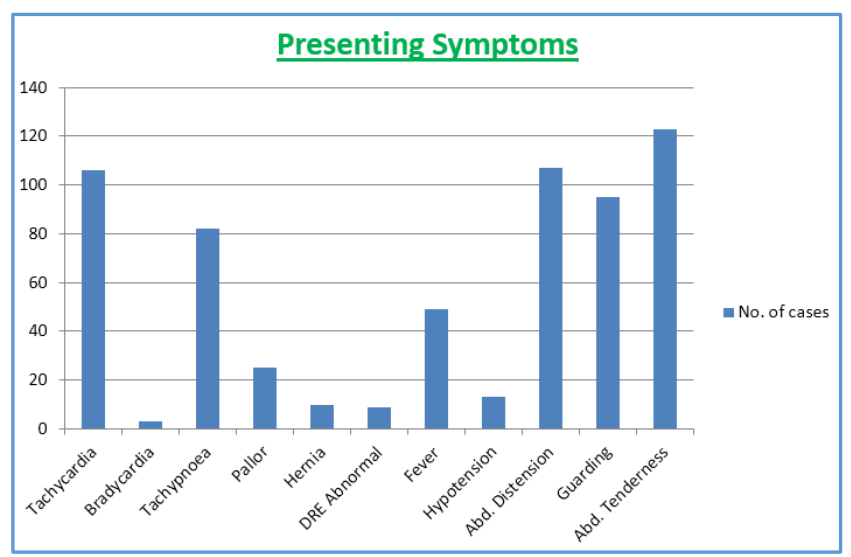

Figure 1. Clinical Features of the Patients at Presentation

\section{Investigations}

About $32.8 \%$ of cases (45) were having Haemoglobin (Hb) of less than 10 grams per decilitre of blood $(\mathrm{g} / \mathrm{dL})$ and $59.1 \%$ of cases (81) had their Total White Blood Cell count (CBC) of $>11,000$ cells per cubic millimetre (cumm) of blood. Renal function was abnormal in $50.4 \%$ (69) of cases.

Plain abdominal x-ray was done in all but 2 cases, because of their haemodynamic instability. 65 cases (48.1\%) had free gas under the diaphragm and 26 cases had multiple air fluid levels (19.2\%), whereas in 21 patients $(15.5 \%)$ there were no specific findings. A schematic diagram of various plain abdominal $x$-ray findings is given in Figure 2.

Ultrasound (USG) of the abdomen was done in about $42.3 \%$ (58) of cases, out of which 29 cases $(21.2 \%$ of total laparotomy cases) shows features suggestive of intestinal obstruction and 18 cases (13.1\%) showed free fluid in the abdomen. In 9 cases (6.5\%), solid organ injury was identified.

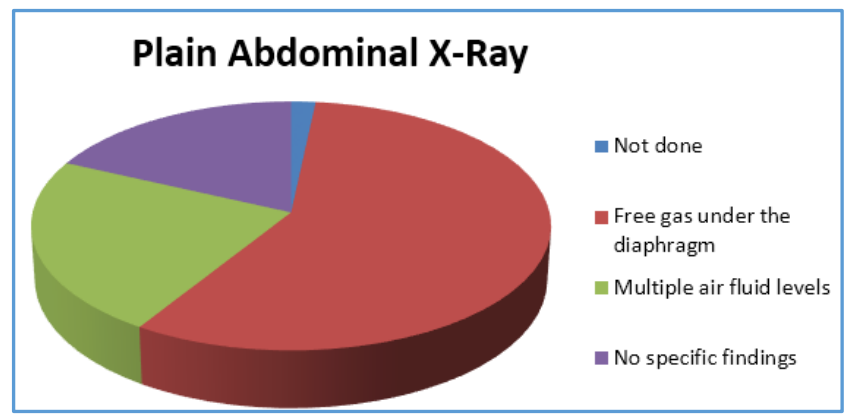

Figure 2. Percentage distribution of Plain Abdominal X-Ray Findings

Contrast-Enhanced Computed Tomography of abdomen was done in a total of $22(16.1 \%)$ cases. It was done in case of doubtful or incomplete diagnosis, especially in blunt trauma abdomen cases. Some cases in which gas under the diaphragm was not detected on Plain Abdominal X-ray were detected on CECT Abdomen.

\begin{tabular}{|c|c|c|}
\hline CECT Abdomen Findings & Frequency & Percent \\
\hline Not done in, & 115 & 83.9 \\
Features suggestive of perforation & 6 & 4.4 \\
Features suggestive of intestinal & & \\
obstruction & 8 & 5.8 \\
Free fluid in the peritoneum & 2 & 1.5 \\
Splenic injury found in, & 2 & 1.5 \\
Abscess and Collections & 3 & 2.2 \\
No abnormality detected & 1 & 0.7 \\
Total & $\mathbf{1 3 7}$ & $\mathbf{1 0 0}$ \\
\hline Table 1. Contrast-Enhanced Computed Tomography \\
(CECT) Abdomen Findings of the Patients \\
\hline
\end{tabular}

After the establishment of diagnosis which mandated a laparotomy, patient was taken to operation theatre as soon as possible. In the overwhelming percent of patients (78.1\%), laparotomy was done between 8 - $24 \mathrm{hrs}$. after the arrival at casualty. Whereas in 21 cases, the decision to 'open abdomen' was taken after 24 hours.

\begin{tabular}{|c|c|c|}
\hline Postoperative Diagnosis & $\begin{array}{c}\text { Frequen } \\
\text { cy }\end{array}$ & \% \\
\hline Gastric perforation & 9 & 6.6 \\
Duodenal perforation & 54 & 39.4 \\
Ileal perforation & 3 & 2.2 \\
Jejunal perforation & 1 & 0.7 \\
Appendicular perforation & 3 & 2.2 \\
Large bowel perforation & 2 & 1.4 \\
Gall bladder perforation & 1 & 0.7 \\
Large bowel obstruction & 5 & 3.7 \\
Small bowel obstruction & 17 & 12.4 \\
Obstructed inguinal hernia & 1 & 0.7 \\
Obstructed Umbilical hernia & 2 & 1.4 \\
Obstructed incisional hernia & 1 & 0.7 \\
Blunt liver trauma & 6 & 4.4 \\
Blunt splenic injury & 11 & 8.0 \\
Blunt small bowel injury & 5 & 3.7 \\
Blunt large bowel injury & 2 & 1.4 \\
Blunt bladder injury & 1 & 0.7 \\
Blunt mesenteric injury & 2 & 1.4 \\
Penetrating injury stomach & 2 & 1.4 \\
\hline
\end{tabular}




\begin{tabular}{|c|c|c|}
\hline Penetrating injury small bowel & 1 & 0.7 \\
Ruptured liver abscess & 3 & 2.2 \\
Mesenteric ischaemia & 2 & 1.4 \\
Re-laparotomy for anastomotic leak & 2 & 1.4 \\
Post coloanal anastomosis wound dehiscence & 1 & 0.7 \\
Total & $\mathbf{1 3 7}$ & $\mathbf{1 0 0}$ \\
\hline \multicolumn{2}{|c|}{ Table 2. Frequency Distribution based } \\
on Postoperative Diagnosis
\end{tabular}

In the postoperative period, $31.4 \%$ of cases (43 cases) required Intensive Care Unit (ICU) admission. Some patients were on ventilator, others were admitted to correct postoperative hypotension with inotropic support, while some others were admitted for intensive monitoring after a major case.

\section{Postoperative Complications}

Total no. of patients developing complications (77) $=56.2 \%$, the details of which is given below in the Table 3 .

\begin{tabular}{|c|c|c|}
\hline & Frequency & Percent \\
\hline Wound infection & 37 & 27 \\
Wound dehiscence & 2 & 1.46 \\
Intra-abdominal abscess & 3 & 2.2 \\
Postoperative bleeding & 6 & 4.4 \\
Enterocutaneous fistula & 9 & 6.57 \\
Deep Venous Thrombosis & 10 & 7.3 \\
Pneumonia & 8 & 5.84 \\
Acute Coronary Syndrome & 2 & 1.46 \\
No complications & 60 & 43.8 \\
Total & $\mathbf{1 3 7}$ & $\mathbf{1 0 0 . 0}$ \\
\hline Table 3. Postoperative Complications of the Patients \\
\hline
\end{tabular}

Majority of postoperative complications (27\%) was wound infection. Four cases developed transient anastomotic leak which settled spontaneously in 3 - 4 days, whereas 5 other cases developed Enterocutaneous fistula, which required extended period of admission and management. 20 patients $(14.6 \%)$ died in the postoperative period, during the initial hospital stay. Mortality in non-trauma group was maximum among the elderly, and in those with large bowel perforation and in patients with extensive bowel ischaemia due to mesenteric ischaemia. In the trauma group, severe haemorrhagic shock and major organ or multiple solid organ injuries and/or great vessel injuries were the cause of death.

\section{DISCUSSION}

Emergency laparotomies are one of the most common surgeries, which are performed in an operating room. This procedure is done mainly for two broad category of aetiologies- 1) Acute Abdomen and 2) Trauma.

Previously, one of the most hazardous of surgeries used to be trauma abdomen. With the advent of various diagnostic modalities we can now select and chose patients with trauma, which and when to open. It is found to be safe to avoid a laparotomy in the trauma patients, who are haemodynamically stable and in whom bowel is not perforated. Many of such patients like those with lower grade splenic and hepatic injuries do not require surgery and they recover well conservatively.

Many a times, the exact diagnosis will not be certain before the surgery and the pathology is identified only after opening the abdomen. Such laparotomies are called as
'Exploratory laparotomy.' The main reason for not reaching a definitive diagnosis preoperatively is the emergency nature of the condition, where a balance between exhaustive investigation and potential or actual deterioration of patient's condition is to be created.[1] On the other hand, a short delay in resuscitating a severely shocked patient or a patient in septic shock and also some delay in which basic investigations are done will in fact benefit the patient.[1]

The most significant accomplishment of modern surgery is making surgery comparatively safe as compared to the previous times.[3] Safety should be our main concern, as our treatment should not be more hazardous than the disease or the pathology itself. Next to safety, our aim of the surgery should be to remove or correct the causative factor with as minimum a trauma as possible and restore the body function and ultimately cure the patient. With this goal in mind, optimisation of the patient without undue delay leads to a better outcome following surgery.

Hypovolemic shock is a major cause of death in patients with abdominal trauma. And the most easy and quick way to measure the circulatory state is by monitoring the pulse rate and blood pressure.[4] Focused abdominal sonography in trauma (FAST) is being done routinely as a diagnostic tool to detect free fluid in the abdominal cavity. This has helped immensely in the decision making process. [5]

According to Pfeifer et al, there is a sharp decrease in the prevalence of early death due to haemorrhage from $25 \%$ to $15 \%$ during the past decade.[6] This happy outcome may be due to various reasons, but it is more apparent that this improvement in the trauma mortality could be due to widespread implementation of the Advanced Trauma Life Support (ATLS) principles and also due to the concept of damage control surgery.[4] Damage control surgery as a principle and as a concept was introduced by Rotondo et al in 1993. It is based on a staged surgical approach in dealing with the patients with trauma. It is a method to deal with the condition or the pathology, which is currently killing the patient and ignoring for time being or postponing the definitive surgical management only after the patient tides over the present situation.

Patient with severe injuries requiring operative interventions are associated with an interlinked triad of hypothermia, acidosis and coagulopathy. This is also called as the lethal triad. Each of the three components of this triad stimulates the other two, creating a vicious cycle which can lead to death if it is not broken.[7] One of the main principles of the damage control surgery is to break this vicious cycle of death. To achieve this goal, damage control surgery is done in three phases. In the first phase, the aim is to quick control bleeding and contamination. This is done by rapid surgical steps like packing, ligation of bleeders, vessel shunting and resection of diseased and damaged bowel, without anastomosis. In the second phase patient is shifted to a Critical Care Unit for resuscitation, warming and correction of coagulopathy (if present). And during the final phase, patient is taken back to the operating room for definitive repair and also to deal with the unfinished job of reconstituting the vascular and bowel continuity and/or resection of the injured organs, unpacking and closing the abdomen, because in almost every abdominal trauma patient is managed by damage control surgery and the abdomen is left open at the end of the first operation.[7] 


\section{Acute Abdomen}

'Acute abdomen' is a term which is used to include a variety of conditions requiring hospital admission, investigation and treatment. It encompass a whole spectrum of diseases, which ranges from very trivial to many a times life-threatening conditions of surgical, medical and gynaecological specialities.[8] About $50 \%$ of general surgical admissions done under emergency basis are 'Acute abdomen' cases. Common surgical conditions which present with 'Acute abdomen' includes acute appendicitis, acute cholecystitis, gastrointestinal perforation, acute pancreatitis, bowel obstruction, malignant conditions etc. [9]

Among the conditions requiring emergency laparotomy, gastrointestinal perforation causes considerable mortality. The mortality is proportional to peritoneal contamination, site, size and time elapsed between perforation and presentation at the casualty. Other factors affecting the mortality are the physiologic state of the patient, time from last meal, mechanical bowel preparation, any significant coexistent disease, any accompanying bowel obstruction etc. [10-12]

Among these factors, the anatomic site of perforation is the most important, because of the type and burden of enteric contamination. The colonisation of the enteric bacteria progressively increases from stomach (less than $10^{3}$ organisms per gram of luminal contents) due to the presence of hostile local environment (due to acidic, biliary and pancreatic secretions), which inhibits the growth of bacteria. And when it comes to the jejunum the bacterial content increases to $10^{4}$ per gram and progressively increases to $10^{12}$ per gram of luminal contents in case of colon.[11,12]

The bacterial load is inversely proportional to the relative chemical toxicity of the luminal fluid composition. Therefore, the perforations of the proximal bowel, although presenting dramatically and many a times with sudden clinical deterioration, yet such cases present early, bacterial contamination is less and are often managed before frank sepsis ensues. Conversely, the distal bowel perforations are chemically neutral (hence present late), but these are microbiologically very dangerous. This combination of late presentation and bacterial contamination often turns out to be lethal for the patients. $[9,13,14]$

A meticulous history taking and proper physical examination is very crucial to determine the seriousness of presentation, identify surgical emergencies, evaluate surgical risk, guide in targeted diagnostic testing and preoperative optimisation of the individual patient. [13,15,16]

Radiographic examination of abdomen is one of the most important and many a times the only special investigation required to arrive at a working diagnosis of a patient presenting with 'Acute abdomen.' The presence of free subdiaphragmatic air on an upright chest and abdominal radiograph indicates bowel perforation. Although, a good quality upright chest radiograph can detect as less as $1 \mathrm{ml}$ of free gas below right hemidiaphragm, the sensitivity is only $50 \%-70 \%$. Another drawback is the site of perforation could not be elucidated on plain upright chest and abdominal radiograph.[17]

Among other modalities of investigation, ultrasound has an advantage of no radiation exposure, but its utility as a definitive investigation to exclude pneumoperitoneum is not clear. Computed tomography (CT) has shown some accuracy $(86 \%)$ in predicting the site of perforation. ${ }^{[14,18]}$

The broad outline of treatment, when gastrointestinal perforation is identified or very much suspected is fluid resuscitation, antibiotics, control of source, support of organ system and maintaining nutrition of the patient.[14,18] Among the aetiologies, intestinal obstruction presents with a unique challenge in the diagnosis and management, as a delay in diagnosis and inappropriate management may lead to the death of the patient. About $80 \%$ of all intestinal obstructions are due to large bowel obstruction. Management of intestinal obstruction includes giving proper analgesia, decompression of intestine by nasogastric aspiration, correction of fluid and electrolyte derangement and surgery where appropriate.[19]

In cases of adhesive obstruction of small intestine, majority of cases settle with conservative management, hence this line of management should be attempted initially if there are no overt signs of strangulation. If there is a primary underlying disease causing obstruction like complicated hernia or when there are signs of peritoneal irritation or when the obstruction failed to settle within 48 hours, then prompt operative intervention is mandatory.[19]

\section{CONCLUSION}

'Acute Abdomen' is the most common indication for emergency laparotomies. Among these cases of 'Acute Abdomen,' perforation peritonitis is the most common indication for a laparotomy. Although, the most common perforation in our study was duodenal perforation, the diagnosis-specific mortality was higher among large bowel perforations and mesenteric ischaemia cases. In laparotomies due to trauma, extensive solid organ and/or great vessel injuries was the major cause of death. A large number of laparotomies (78.1\%) were performed between 8 hours to 24 hours of presentation at casualty, but many cases $(15.3 \%)$ were also performed after 24 hours due to various factors, some of which can be improved upon. Our institution is working on it to improve on this account.

\section{REFERENCES}

[1] Rintoual R. Farquharson's textbook of operative surgery. $7^{\text {th }}$ edn. Edinburgh, London: Churchill Livingstone 1986.

[2] Squires RA, Postier RG. Acute abdomen. In: Towsend CM, Beauchamp RD, Evers BM, et al. eds. Sabiston textbook of Surgery: the biological basis of modern surgical practice. 19th edn. Philadelphia: Elsevier 2012: p. 1141-59.

[3] Deaver JB. When and when not to open the abdomen in acute surgical conditions. Ann Surg 1929;89(3):340-53.

[4] Lund H, Kofoed SC, Hillingso JG, et al. High mortality after emergency room laparotomy in haemodynamically unstable trauma patients. Dan Med Bull 2011;58(5):A4275.

[5] Farahmand N, Sirlin CB, Brown MA, et al. Hypotensive patients with blunt abdominal trauma: performance of screening US. Radiology 2005;235(2):436-43.

[6] Pfeifer R, Tarkin IS, Rocos B, et al. Patterns of mortality and cause of death in polytrauma patients--has anything changed? Injury 2009;40(9):907-11. 
[7] George C, Velmahos M, Hasan B, et al. Damage control operation. Current surgical therapy. $10^{\text {th }}$ edn. 2011: p. 970-4.

[8] Gordon PH, Nivatvongs S, Mulholland MW. Principles and practice of surgery for colon, rectum and anus. Shock 1999;12:328.

[9] Nathens AB, Rotstein OD. Therapeutic options in peritonitis. Surg Clin North Am 1994;74(3):677-92.

[10] Putcha RV, Burdick JS. Management of iatrogenic perforation. Gastroenterol Clin North Am 2003;32(4):1289-309.

[11] Guarner F, Malagelada JR. Gut flora in health and disease. Lancet 2003;361(9356):512-9.

[12] Guarner F. Enteric flora in health and disease. Digestion 2006;73 (Suppl 1):5-12.

[13] Martin RF, Rossi RL. The acute abdomen. An overview and algorithms. Surg Clin North Am 1997;77(6):122743.
[14] Fultz PJ, Skucas J, Weiss SL. CT in upper gastrointestinal tract perforations secondary to peptic ulcer disease. Gastrointest Radiol 1992;17(1):5-8.

[15] Lyon C, Clark DC. Diagnosis of acute abdominal pain in older patients. Am Fam Physician 2006;74(9):1537-44.

[16] Gerhardt RT, Nelson BK, Keenan S, et al. Derivation of a clinical guideline for the assessment of nonspecific abdominal pain: the Guideline for Abdominal Pain in ED setting (GAPEDS) Phase 1 Study. Am J Emerg Med 2005;23(6):709-17.

[17] MacKersie AB, Lane MJ, Gerhardt RT, et al. Nontraumatic acute abdominal pain: unenhanced helical CT compared with three-view acute abdominal series. Radiology 2005;237(1):114-22.

[18] Urban BA, Fishman EK. Tailored helical CT Evaluation of acute abdomen (CME available print version and on RSNA link). Radiographics 2000;20(3):725-49.

[19] Macutkiewicz C, Carlson GL. Acute abdomen: intestinal obstruction. Surgery (Oxford) 2005;23(6):208-12. 